Classification

Physics Abstracts

$71.30-71.55 \mathrm{~J}-73.60 \mathrm{D}$

\title{
Interplay between microscopic and macroscopic disorder in percolating Pd films
}

\author{
N. Papandreou and P. Nédellec \\ Centre de Spectrométrie Nucléaire et Spectrométrie de Masse, $\mathrm{IN}_{2} \mathrm{P}_{3}-\mathrm{CNRS}, 91405$ Campus \\ Orsay, France
}

(Received 9 September 1991, accepted in final form 21 January 1992)

\begin{abstract}
Thin metallic Pd films (100 $\AA$ ) were submitted to irradiation with $100 \mathrm{keV} \mathrm{Xe} \mathrm{ions.}$ After an initial increase of the atomic disorder, a decrease of the metallic coverage occurs as holes are created due to sputtering. This leads to a percolating transition. The modifications of the electronic mean free path, film thickness and metallic surface coverage were measured in situ up to the percolation threshold by means of resistive measurements, Rutherford Backscattering and Transmission Electron Microscopy experiments. The resistance at $300 \mathrm{~K}$ is found to diverge near the percolation threshold with the typical $2 \mathrm{D}$ exponent value. A phenomenological model accounts for the evolution of the topological parameters as the fluence (number of incoming ions per unit surface) increases. We present low temperature electrical measurements on films irradiated at different fluences, thus lying at different distances from the percolation threshold. Our results show a strong interaction between the microscopic and the macroscopic disorder near the percolation threshold: the conductance temperature dependence crosses over from a logarithmic shape (i.e. characteristic of weak localization in 2D metallic films) to a power law dependence, due to the non-Euclidean (fractal) dimensionality of the percolating metallic structure. Moreover, very close to the threshold but always above it, the conductance varies exponentially with temperature, revealing the appearance of an Anderson insulator, although a continuous metallic path exists across the samples. At $4.2 \mathrm{~K}$, the resistance approaches the percolation threshold with exponent 2, (different from the classical percolation value 1.3 observed at $300 \mathrm{~K}$ ). This new exponent must contain information on the observed Metal-Insulator Transition.
\end{abstract}

\section{Introduction.}

Disorder greatly influences the electrical transport properties of a metal and may induce a Metal-Insulator Transition (M.I.T.). Quantum localization [1-3] and classical percolation [4, 5] induce two types of M.I.T. as the disorder appears at two different scales : a microscopic (atomic disorder) and a macroscopic (granular disorder) one.

As long as one considers « dilute » disorder in a pure metal characterized by the presence of atomic defects (vacancies, interstitials, ...) in the crystal lattice, the classical picture of uncorrelated collisions between the electrons and the randomly distributed defects success- 
fully describes the conductance of a metal at low temperature. With increasing disorder, new effects appear that are explained by the theory of quantum localization, which takes into account the conservation of the phase coherence of an electronic wave function between two inelastic collisions. When the microscopic resistance exceeds a critical value, all the electrons become localized at zero temperature : this disorder-induced M.I.T. is called the Anderson transition.

Macroscopic disorder, introduced for instance by the presence of randomly distributed metallic and insulating grains (or holes as in our experiment) of typical size 100 to $1000 \AA$, can lead to a percolating structure. The relevant parameter in the case of a $2 \mathrm{D}$ film is the metallic surface coverage $p$. As long as $p$ is larger than a critical value $p_{c}$, the percolation threshold, a continuous metallic path exists. In the limit where there are relatively few insulating inclusions, effective-medium theories [6] describe the electrical transport properties but they fail when $p$ approaches $p_{c}$. The geometrical theory of percolation is designed for this régime : it considers the approach to discontinuity as a critical phenomenon and reveals the non-Euclidean (fractal) geometry of the conducting metallic cluster near $p_{\mathrm{c}}$.

Considerable theoretical and experimental work has been devoted separately to each kind of disorder and has explained successfully their respective effects. Only a few studies, however, have focused on the problem of electrical transport when both kinds of disorder coexist in the same sample. Theoretical analyses [7-13] have treated the role of the macroscopic disorder in the vicinity of the Anderson transition and the main prediction is that the electrical properties of a metal are not obtained by a simple superposition of the percolation and localization effects. Some experimental studies [14-17] have shown that the percolation structure enhances the Anderson localization phenomena near the percolation threshold.

Our goal has been to study systematically the low temperature electrical transport in a system combining both aspects of disorder at varying amounts; we determine the conditions under which percolation and localization are no longer independent but interact and give rise to new transport laws.

The paper is structured as follows : section 2 recalls theoretical predictions on percolation, localization and their interaction. The sample preparation technique is presented in section 3. We have used ion irradiation to create both atomic defects and holes in thin Pd films. The characterization experiments are described and the parameters, which are important for the disorder, i.e. the bulk electronic mean free path (m.f.p.) $\ell_{\mathrm{b}}$, the thickness $d$ and the surface coverage $p$ are measured at different fluences. This is done by Transmission Electron Microscopy (T.E.M.) and Rutherford Backscattering (R.B.S.) experiments [18], combined with the study of the resistance at $300 \mathrm{~K}$, which depends only on the film topology near the percolation threshold [19]. Then, we present the conductance and magnetoconductance measurements performed in the liquid helium temperature range (Sect. 4). The low temperature conductance is sensitive to both the topology and the atomic disorder, allowing us to study the interference between their respective effects. Finally, we draw a phase diagram in the plane defined by the bulk mean free path $\ell_{\mathrm{b}}$ and the metallic surface coverage $p$. This phase diagram covers the homogeneous régime $(p=1)$ as well as the vicinity of the percolation threshold $\left(p \simeq p_{c}\right)$.

\section{Theoretical background.}

2.1 MACROSCOPIC DISORDER AND PERCOLATION [4]. - Although percolation theory was originally a mathematical concept, it has been applied to the description of many physical problems and, in particular, to the morphological analysis of inhomogeneous films [5]. In this case, the important parameter is the metallic surface coverage $p$. If $p$ exceeds the percolation 
threshold $p_{c}$, there is a continuous metallic path connecting one end of the film to the other ( « the infinite cluster »). There are two characteristic scales for a percolating system: the grain scale $\xi_{0}$ and the percolation length $\xi_{\mathrm{p}}$ which follows a power law near $p_{\mathrm{c}}$.

$$
\xi_{\mathrm{p}} \approx \xi_{0}\left(p-p_{\mathrm{c}}\right)^{-\nu}
$$

The exponent $\nu$ is equal to $4 / 3$ in $2 D$. For $p>p_{c}, \xi_{\mathrm{p}}$ can be interpreted as the scale above which the system is homogeneous and presents a constant mass density and for $p<p_{\mathrm{c}}$, it is basically the mean linear size of the clusters.

The most spectacular consequence of the randomness of the infinite cluster is the scale dependence of its physical properties. Let us consider electrical transport : at $p_{\mathrm{c}}$, the conductivity $\sigma$ vanishes according to a power law characterized by exponent $\mu$ (at 2D, $\mu=1.3)$ :

$$
\sigma \approx\left(p-p_{\mathrm{c}}\right)^{\mu} \quad p>p_{\mathrm{c}}
$$

The conductivity is scale-dependent below $\xi_{\mathrm{p}}[4]$ :

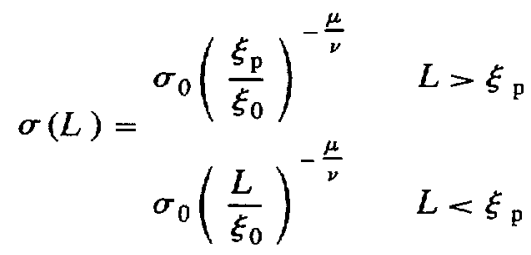

where $\sigma_{0}$ measures the conductivity on the relevant scale (governed by electron diffusion as we will see later).

In $2 \mathrm{D}$, the parameter which describes the intrinsic electrical properties of a material, getting rid of sample geometry effects is $C_{\square}$, the conductance per square (equal to the product of the conductivity with the thickness $d$ ). As the measurement scale is always larger than $\xi_{\mathrm{p}}, C_{\square}$ scales like the conductivity and depends only on $\xi_{\mathrm{p}}$ following :

$$
C_{\square}=\sigma(L) d\left(p-p_{\mathrm{c}}\right)^{\mu}=C_{\square}(L)\left(p-p_{\mathrm{c}}\right)^{\mu} \quad p>p_{\mathrm{c}} .
$$

In this equation, $L$ is a suitable scale, related to electron transport length, which is not necessarily the grain size. The factor $\left(p-p_{c}\right)^{\mu}$ can be viewed as a geometrical form factor: the real length of the tortuous metallic cluster is much larger than the macroscopic length of the sample and the opposite is true for the width. Thus, a percolating sample presents a reduced apparent conductivity with respect to a homogeneous one, with the same microscopic disorder.

2.2 MICROSCOPIC DISORDER AND LOCALIZATION [1]. - In a microscopically disordered homogeneous metal quantum interference and electron-electron interaction effects dominate the conductivity at low temperature because they need phase coherence (i.e. elastic collisions) to become effective [2, 20]. These effects tend to weaken conduction. If, as disorder increases, the elastic m.f.p. is reduced down to the order of the interatomic distance, the electrons become localized and the system behaves as an insulator on the macroscopic scale, even when the density of states at the Fermi level is non-zero : this is the Anderson M.I.T. [1].

The characteristic scale for localization is given by the inelastic length $L_{\phi}$ which is approximatively the mean distance between two inelastic collisions and depends on temperature :

$$
L_{\phi} \approx T^{-\alpha / 2}
$$


The exponent $\alpha$ depends on the inelastic collision mechanism [21, 22] (electron-phonon, electron-electron). The inelastic collisions destroy phase coherence on scales larger than $L_{\phi}$ and localization effects become more efficient at low temperature as $L_{\phi}$ increases.

On the metallic side of the Anderson transition, the weak localization régime, the diffusive motion of the electrons is modified by the interference and the interaction effects. The correction to the conductance is given, at low temperature, as $[3,23]$ :

$$
\Delta C_{\square}(T)=-s \frac{\alpha e^{2}}{2 \pi^{2} \hbar} \ln \left(\frac{T}{T_{0}}\right)+\frac{e^{2}}{2 \pi^{2} \hbar}\left(2-\frac{3}{2} F\right) \ln \left(\frac{k_{\mathrm{B}} T \tau_{0}}{\hbar}\right) .
$$

The first term corresponds to the interference effects and is positive or negative, depending on the value of the phenomenological parameter $s$ related to the spin-orbit (SO) interaction : $s$ varies from -1 in the weak SO limit to $1 / 2$ in the strong SO limit. The second term gives the interaction contribution controlled by the screening factor $F$ which goes to zero in a highly disordered metal where screening is weak.

A magnetic field destroys the phase coherence and also strongly disturbs the quantum interference. In order to describe its effects, one associates to each collision length a characteristic magnetic field. For example, the inelastic field $H_{\phi}$ is related to the inelastic length by the relation :

$$
H_{\phi} L_{\phi}^{2}=\frac{\hbar}{4 e}
$$

The magnetoconductance (MC) is positive in the weak SO limit and negative in the strong $\mathrm{SO}$ limit. In the intermediate case, $\mathrm{MC}$ is negative at low temperature and positive at high temperature.

The measure of both $C_{\square}(T)$ and $C_{\square}(H)$ permits a complete description of the weak localization effects.

On the insulating side of the Anderson transition, the electronic transport is achieved by thermally activated hopping between localized states. The conductance varies exponentially with temperature :

$$
C_{\square}(T) \approx \exp \left(-\left(\frac{T_{1}}{T}\right)^{\gamma}\right)
$$

$T_{1}$ is a constant, generally related to the spatial extention $\xi_{\ell}$ of a localized state [24]. The value of the exponent $\gamma$ depends on the hopping mechanism [24]: $\gamma=1$ for nearest-neighbour hopping [25], $\gamma=\frac{1}{d+1}$ when the tunnel effect is important (the variable range hopping where $d$ is the Euclidean dimensionality) [26] and $\gamma=1 / 2$ when the electron-electron interactions predominate [27].

The Anderson transition may also be obtained by varying the temperature, keeping the disorder constant [28]: the nature of the electronic states depends on the competition between $\xi_{\ell}$ and $L_{\phi}$. as long as $\xi_{\ell}<L_{\phi}$, the electrons are localized and the inelastic collisions induce thermally activated transitions between localized states. With increasing temperature, we may have $\xi_{P}>L_{\phi}$. an inelastic collision occurs before the elastic ones succed in « establishing» a localized state. The electronic states become extended: an Anderson insulator becomes a metal at high temperature.

2.3 THE PERCOLATION-LOCALIZATION CROSSOVER. - Since all localization effects take place within a scale $L_{\phi}$ while in a percolating system the homogeneity scale is given by the 
percolation length $\xi_{\mathrm{p}}$, localization effects in a percolating régime will depend on the interplay between the different characteristic lengths. We distinguish two cases $[9,10]$ :

i) $L_{\phi}<\xi_{0}$ (high temperature) or $L_{\phi}>\xi_{\mathrm{p}}$ (low temperature). The weak localization corrections are those of the homogeneous limit (Eq. (6)) and the percolation structure merely introduces a correction to the geometrical form factor. We get :

$$
\Delta C_{\square}(T) \approx\left(p-p_{c}\right)^{1.3} \ln (T)
$$

In this limit, we have a superposition of the percolation and localization effects.

ii) $\xi_{0}<L_{\phi}<\xi_{\mathrm{p}}$ (« intermediate » temperature). This is the inhomogeneous régime and Gefen et al. $[9,10]$ have derived for weak localization on a percolation fractal (in 2D) the following temperature dependence for the conductance :

$$
\Delta C_{\square}(T) \approx\left(p-p_{c}\right)^{1.3} T^{-0.34 \alpha}
$$

This expression recalls the $1 \mathrm{D}$ homogeneous case because the effective dimensionality is between 1 and 2 .

Just above the percolation threshold $\xi_{\mathrm{p}}$ is large and, even at low temperature, we get the inhomogeneous régime. It has been shown $[9,10]$ that the scale-dependent conductivity goes to zero at large scales. This means the system behaves macroscopically as an insulator, even if it is weakly disordered at the microscopic scale. The percolating geometry acts as an « amplifier» of the microscopic disorder.

Therefore, the analysis above may be reconstructed as follows. Let us imagine an inhomogeneous system in which microscopic disorder is weak and constant; allow the temperature to vary very little, so that $L_{\phi}$ is constant. When we reduce the surface coverage $p$ to approach the percolation threshold, we shall encounter the following situations :

- far from the threshold $\xi_{0}<\xi_{p}<L_{\phi}$. the system is a metal. The conductance is given by (9) where the effects of percolation and localization are independent ;

- near the threshold $\xi_{0}<L_{\phi}<\xi_{\mathrm{p}}$. the system is still a metal. The conductance is given by (10). The effects of percolation and localization interfere ; the theory of weak localization takes into account the anomalous diffusion of the electrons in the fractal medium;

- very close to the threshold $\xi_{0}<L_{\phi} \ll \xi_{\mathrm{p}}$. the electronic states become localized and the system behaves as an Anderson insulator. The interference between the two kinds of disorder is strong : it all happens as if the microscopic disorder were increased by orders of magnitude to drive the system across the Anderson transition.

\section{Preparation and characterization.}

Thin Pd films (typically $100 \AA$ ) were deposited under a pressure of $10^{-8}$ Torr by electron gun evaporation on quartz substrates for resistive and R.B.S. experiments, on $\mathrm{NaCl}$ substrates for T.E.M. observations. The substrates are held at room temperature during the evaporation. They had previously been coated with a SiO layer of $150 \AA$, which ensures similar growth conditions, whatever the substrate below SiO. Such Pd films are homogeneous [29]. The samples were irradiated under normal incidence with a $100 \mathrm{keV} \mathrm{Xe}$ ion beam. The initial irradiation current is $500 \mathrm{nA}$ and was reduced to $30 \mathrm{nA}$ in order to avoid local overheating when the samples become inhomogeneous. Four samples could be irradiated simultaneously, with the possibility of covering one, two or three of them. Thus, at each irradiation step, we obtained irradiated samples at four different fluences and this allowed us to cover a large fluence range in a few runs. Irradiations were carried out either at $300 \mathrm{~K}$ or at $4.2 \mathrm{~K}$. In both cases, the resistance was measured in situ after each irradiation step using dc measurements. 
The samples irradiated at room temperature are not stable near the percolation threshold and evolve in time between two irradiation steps. At the end of the irradiation, they were transferred between the implanter and the cryostat. Some of them were measured down to $100 \mathrm{mK}$ in a ${ }^{3} \mathrm{He}-{ }^{4} \mathrm{He}$ dilution cryostat. For low temperature irradiation, the four Pd films were mounted in a $\mathrm{He}$ cryostat [30] coupled to the implanter, allowing a complete in situ study of the temperature and magnetic field dependence of the resistance in the range $1.5 \mathrm{~K}<T<20 \mathrm{~K}$ and $0<H<4$ teslas at each fluence step. Moreover, the low temperatureimplanted percolating samples are stable at low temperature and one can get closer to the percolation threshold.

Structural information is very important in order to understand the electrical properties, especially for the inhomogeneous samples. This information is obtained from R.B.S. experiments, carried out in situ in parallel with resistive measurements during irradiation at 300 K. In situ T.E.M. observations were performed with an electron microscope coupled to the implanter; the samples were tilted at $40^{\circ}$ with respect to the plane perpendicular to the beam. Two series of observations were carried out at $300 \mathrm{~K}$ and $15 \mathrm{~K}$. The figure 1 shows a series of pictures obtained in situ at increasing fluences from (a) to (d) under $300 \mathrm{~K}$

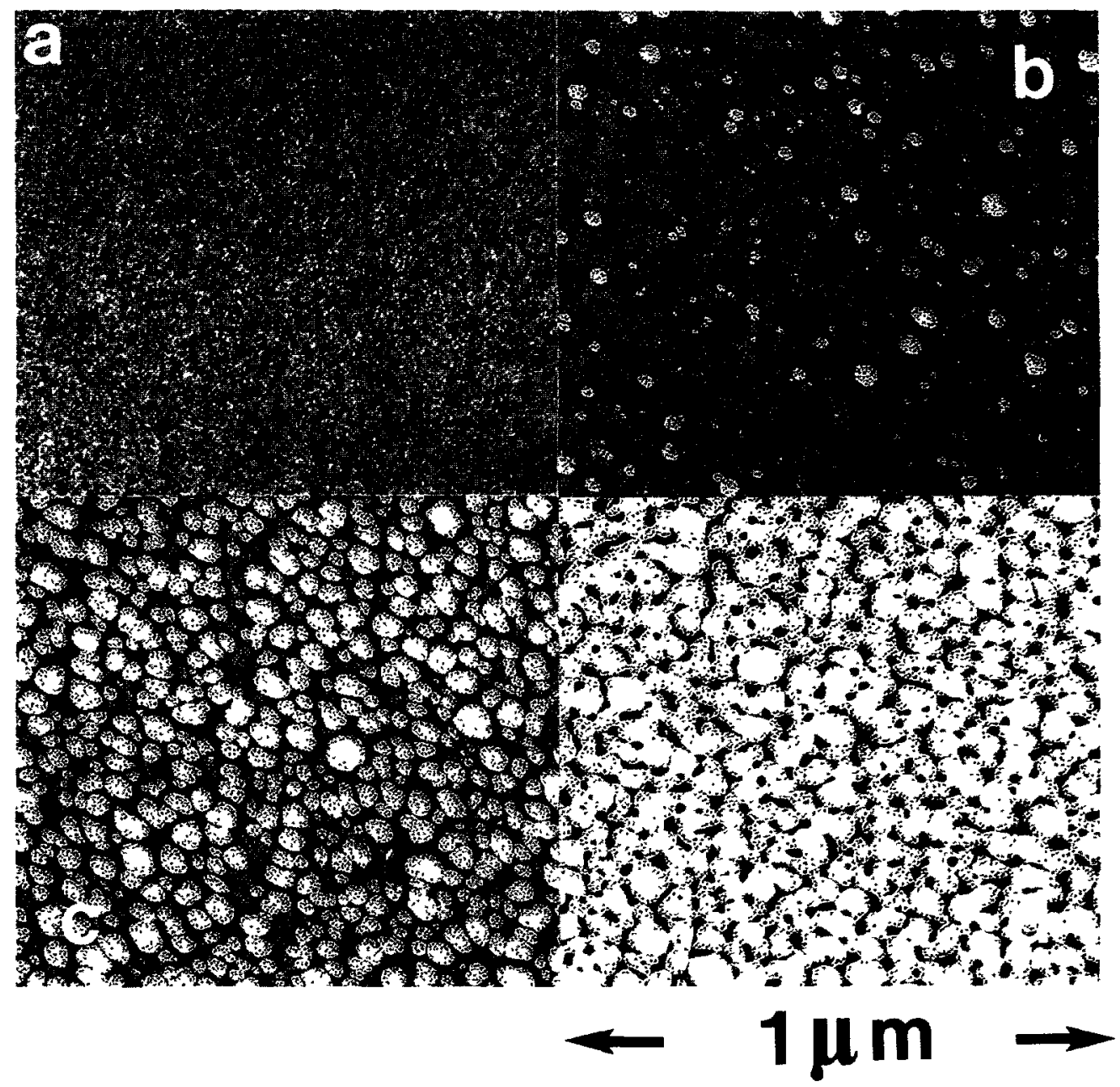

Fig. 1. - T.E.M. pictures of an irradiated Pd film at $300 \mathrm{~K}$ for different Xe fluences. 
irradiation. The Pd regions are dark and the non-irradiated polycrystalline film is shown in (a). Irradiation causes erosion due to sputtering and holes to appear when a critical thickness is reached. The creation of the first holes (b) and the appearance of a percolating structure (c) are visible. Once the percolation threshold is crossed (d), the metallic cluster is disconnected. The T.E.M. observations at $15 \mathrm{~K}$ showed that, under irradiation, the evolution of the metallic surface coverage is the same as in $300 \mathrm{~K}$ irradiation within $10 \%$ for the same fluences.

In our experiments, three parameters are related to the disorder: the bulk mean free path $\mathcal{P}_{\mathrm{b}}$ (or the related resistivity $\rho_{\mathrm{b}}$ ), the thickness $d$ and the metallic surface coverage $p$. Although the measurement of the resistance per square $R_{\square}$ only provides indirect information on these variables, the fluence dependence of its room temperature value gives us a set of simple relations for the evolution of $\rho_{\mathrm{b}}, d$ and $p$ with the fluence $\Phi$. This is possible because the $R_{\square}(\Phi)$ curves display three distinct régimes (I, II and III) as seen in figure 2 . These correspond respectively to atomic defect creation, erosion and hole creation. Our model [19] to analyze the whole $R_{\square}(\Phi)$ curve assumes that each régime is mainly controlled by the variation of a single parameter out of the three. In the first régime, the initial increase of $R_{\square}$ is related to the creation of defects in the bulk and is dominated by the decrease of the electronic m.f.p.; the second régime depends on the thickness modification. The resistance per square $R_{\square}$ of the films, in both régimes, is given by the Fuchs-Sondheimer law [31]:

$$
R_{\square}=\frac{\rho_{\mathrm{b}}}{d}+\frac{3}{8} \frac{\rho_{\mathrm{b}} \ell_{\mathrm{b}}}{d^{2}}
$$

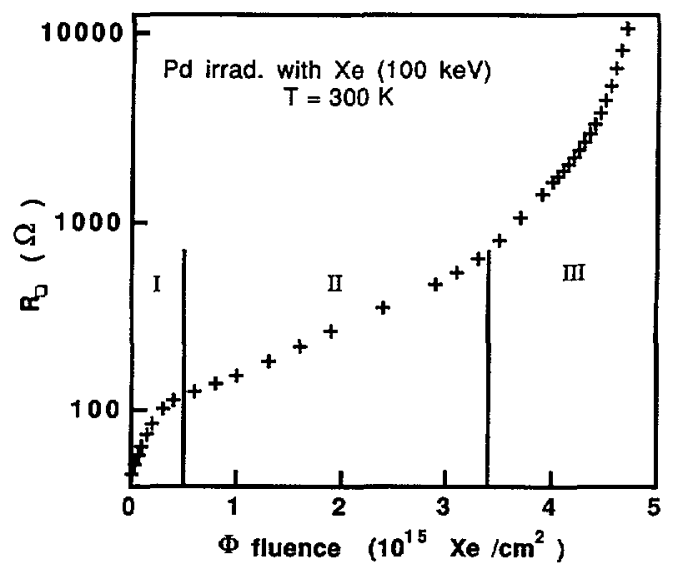

Fig. 2. - Semilog plot of the resistance versus fluence for a Pd film irradiated at $300 \mathrm{~K}$. Three régimes are distinguished : I) bulk defect creation, II) sputtering and III) hole creation.

This relation applies for a homogeneous film (the metallic coverage $p=1$ ), a diffuse surface reflection and $\ell_{\mathrm{b}}<10 \mathrm{~d}$. $\rho_{\mathrm{b}}$ is the resistivity of an equivalent bulk Pd.

At the end of régime $I$, the concentration of bulk defects reaches saturation and atomic sputtering dominates [32]. The change in $R_{\square}$ is then governed by the thickness decrease, via the sputtering yield $Y$, the number of ejected surface atoms per incoming ion. The thickness decrease is given by the differential equation :

$$
\frac{\delta d}{\delta \Phi}=-n Y
$$


where $n$ is the atomic density. $Y$ is proportional to the energy deposited in the target by the ion beam in elastic collisions with the atomic nuclei. In our case, the mean ion range is much larger than the thickness and the incoming ions pass through the film after suffering a number of elastic collisions, roughly proportional to the thickness. In this case, which is different from the "thick sample" in which $Y$ is constant, $Y$ is roughly proportional to $d$ and can be expressed in the form :

$$
\Phi_{d}=\frac{n d}{Y}
$$

$\Phi_{d}$ is a constant with the dimension of a fluence and is characteristic of the material. From equations (12) and (13), we deduce the thickness modification :

$$
d=d_{0} \exp \left(-\frac{\Phi}{\Phi_{d}}\right)
$$

where $d_{0}$ is the initial thickness.

Régime III is dominated by hole creation and the approach to the percolation threshold : the resistance is found to follow the critical law (Fig. 3) :

$$
R_{\square} \approx\left(\Phi_{\mathrm{c}}-\Phi\right)^{-1.3}
$$

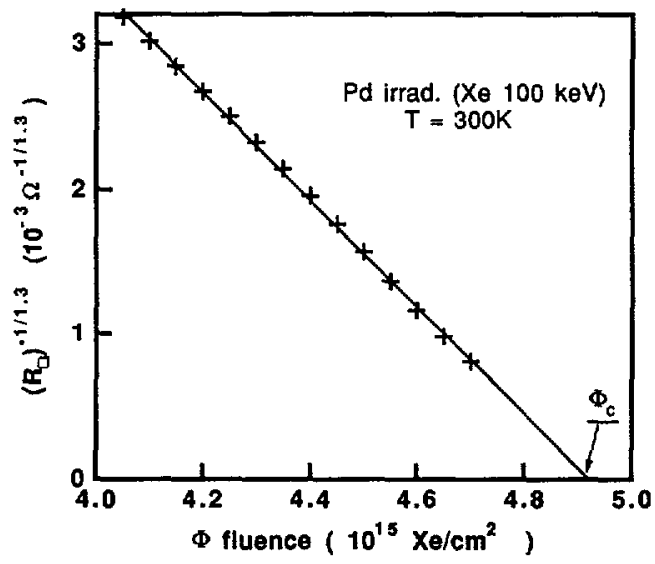

Fig. 3. - Critical behavior of the resistance near the percolation threshold : $R_{\square}$ varies as $\left(\Phi_{\mathrm{c}}-\Phi\right)^{-13}$ The critical fluence $\Phi_{c}$ is obtained by extrapolation at infinite resistance.

The critical fluence $\Phi_{c}$, obtained by extrapolation, corresponds to the percolation threshold : $p_{c}=p\left(\Phi=\Phi_{c}\right)$. The creation of holes is modelized by assuming that the probability of punching a hole is proportional to the available metallic surface and the metallic surface coverage is related to the fluence by an exponential law (Poisson process) :

$$
p=\exp \left\{-\sigma\left(\Phi-\Phi_{1}\right)\right\}
$$

$\sigma$ is the cross section for hole creation and $\Phi_{1}$ the fluence where the first holes appear. In figure 3, for the range considered, $\left(\Phi_{c}-\Phi\right)<0.2 \Phi_{c}$, the expansion of $p(\Phi)$ near $\Phi_{c}$ gives $\left(p-p_{c}\right) \sim\left(\Phi_{c}-\Phi\right)$ whatever the relation between $p$ and $\Phi$; thus, equation (15) corresponds to the theoretical expression (Eq. (4)) in 2D system. 
This model, which explains the $R_{\square}(\Phi)$ curves, has been checked by R.B.S. experiments, in which the thickness and metallic coverage are measured directly at different fluences [18] ; the latter was also measured by means of in situ T.E.M. observations and the deduced values agreed with the R.B.S. results. The experimental values of $d$ and $p$ are compared to relations (14) and (16) and $\Phi_{\mathrm{d}}$ and $\sigma$ are used as adjustable parameters. Theory and experiment agree within $10 \%$. In particular, we calculated the percolation threshold with the result $p_{\mathrm{c}}=0.25 \pm 0.03$, which is an unusually low value for a $2 \mathrm{D}$ percolating system. Our first estimate of the threshold $\left(p_{\mathrm{c}}=0.54\right.$, as reported in Ref. [19]) was obtained by the $R_{\square}(\Phi)$ curve by assuming that $p=1$ at the beginning of the régime III. This is not exact : as shown from T.E.M. observations, the first holes appear before the beginning of the critical behavior of the resistance. As their concentration is small, the resistance is still described successfully by equation (11), valid for a homogeneous system. The crossover to the critical behaviour occurs around $p=0.5$. The value $p_{c}=0.25$ has been confirmed by a preliminary analysis of the T.E.M. pictures (Fig. 1) but we emphasize that we have not been able to perform the morphologic study and the resistive measurements on the same sample. In a number of experiments, important deviations from the theoretical value 0.5 have been found, usually values larger than 0.5 . However, the critical exponent for conductivity is often found close to 1.3 .

Having now measured $R_{\square}(\Phi)$ and $d(\Phi)$, we may evaluate the bulk resistivity $\rho_{\mathrm{b}}(\Phi)$ with the Fuchs' law (Eq. (11)) in régime I, where $p=1$. The result appears in figure 4 for an irradiation at $300 \mathrm{~K}$. We find that $\rho_{\mathrm{b}}$ saturates at the end of the régime $\mathrm{I}$ (the saturation

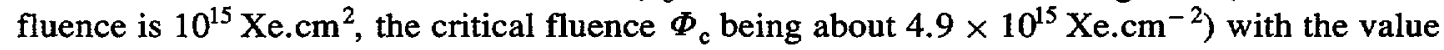
$\rho_{\mathrm{s}}(300 \mathrm{~K})=60 \mu \Omega$.cm. If $\rho_{0}$ is the initial resistivity and $\rho_{\mathrm{i}}$ the irradiation-induced resistivity, $\rho_{\mathrm{s}}=\rho_{0}+\rho_{\mathrm{i}}$. Assuming that $\rho_{\mathrm{s}}$ is the equilibrium value for an irradiated film, for a given fluence $\Phi$, the probability of creating a bulk defect is proportional to the difference between $\rho_{i}$ and the instantaneous resistivity $\rho_{b}(\Phi)$. We obtain an exponential approach of the equilibrium state :

$$
\rho_{\mathrm{b}}=\rho_{0}+\rho_{\mathrm{i}}\left\{1-\exp \left(-\sigma_{1} \Phi\right)\right\}
$$

The solid line in figure 4 represents equation (17), where the cross section $\sigma_{1}$ for the creation of a bulk defect has been used as an adjustable parameter, with the result

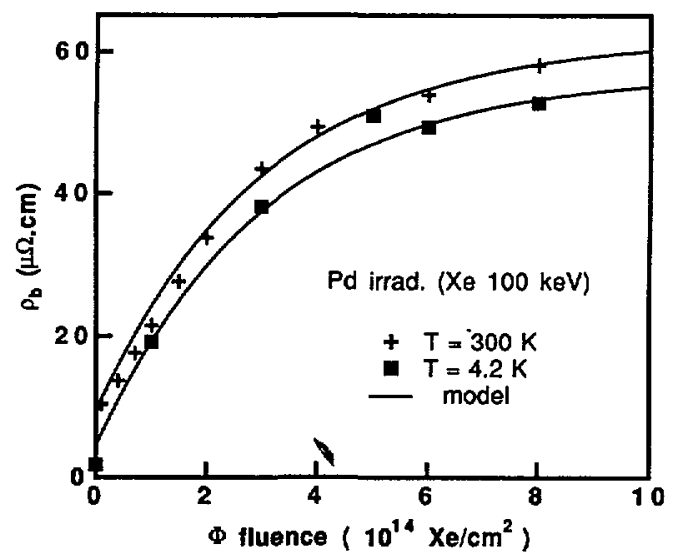

Fig. 4. - Plot of the $300 \mathrm{~K}$ and $4.2 \mathrm{~K}$ bulk resistivity versus fluence (points) and theoretical curves (solid lines), given by equation (17). 
$\sigma_{1}=3.3 \times 10^{15} \mathrm{~cm}^{-2}$ per ion. This value is in agreement with a $T=0 \mathrm{~K}$ calculation assuming Rutherford scattering between incident ions and target atoms.

The low temperature T.E.M. observations showed that under irradiation at $15 \mathrm{~K}$, the evolution of the surface coverage with the fluence is the same as in $300 \mathrm{~K}$ irradiation within $10 \%$. The sputtering yield is temperature independent, hence so is the thickness decrease. Thus, knowing the thickness and having measured $R_{\square}$ at $4.2 \mathrm{~K}$ of an helium temperature irradiated sample, we can calculate $\rho_{\mathrm{b}}$ as a function of fluence. The result is also plotted in figure 4 . The $300 \mathrm{~K}$ and $4.2 \mathrm{~K}$ resistivity-fluence curves are essentially parallel and their difference, of the order of $5 \mu \Omega . \mathrm{cm}$, is comparable to the phonon resistivity of Pd between $4.2 \mathrm{~K}$ and $300 \mathrm{~K}$. For our irradiation conditions, ballistic effects dominate and the disorder created on the microscopic as well as on the macroscopic scale is independent of the irradiation temperature. This property allowed us to prepare samples by $300 \mathrm{~K}$ or $4.2 \mathrm{~K}$ irradiation, the latter giving more stable high-resistance films, lying near the percolation threshold.

The low temperature resistivity $\rho_{s}(4.2 \mathrm{~K})$ is equal to $55 \mu \Omega . \mathrm{cm}$ : using the experimental value [29] $\rho_{\mathrm{b}} \ell_{\mathrm{b}}=4.5 \times 10^{11} \Omega . \mathrm{cm}^{2}$, we find a corresponding bulk elastic m.f.p. of $80 \AA$, which is that of a metal. It is quite clear from this that the electronic m.f.p. is essentially controled by the thickness in régime II. At higher dose, in régime III, we assume that the resistivity $\rho_{\mathrm{s}}$ does not change very much and the above value of the m.f.p. holds up to the percolation threshold. It is well known [34] that, in a pure metal, the damage produced by ion irradiation is subsequently annealed out by the possible recombination processes. It is interesting to note that the size of a conducting channel near the percolation threshold, given by the T.E.M. pictures, is of the order of the bulk m.f.p. This is a favourable situation for an interaction between percolation and localization effects.

\section{Low temperature measurements.}

4.1 Conductance TemPERATURE-DEPENDENCE. - We present our low temperature conductance $C_{\square}(T)$ and magnetoconductance $C_{\square}(H)$ results for a set of irradiated samples and discuss the observed behavior with respect to the theoretical predictions presented above.

The conductance per square temperature curves $C_{\square}(T)$ are shown in figure 5 for samples irradiated at different fluences. The vertical scale corresponds to the variation of the

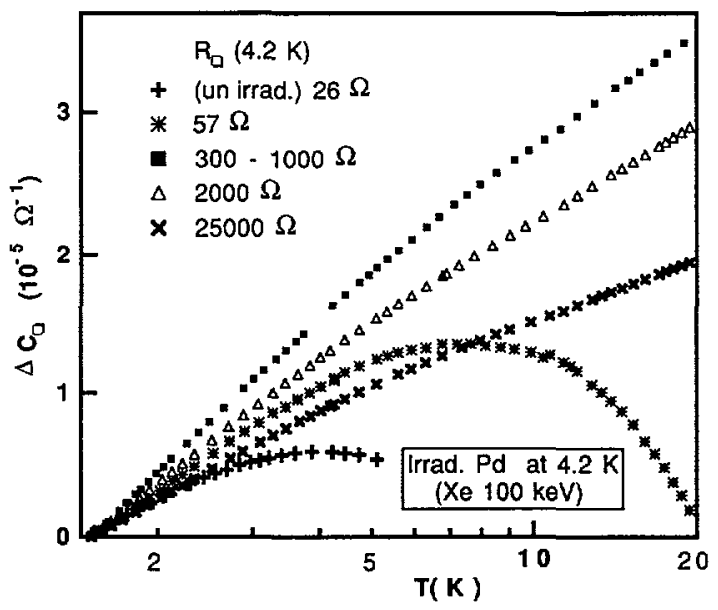

Fig. 5. - Semilog plot of the conductance variation with temperature for different Pd films irradiated at $4.2 \mathrm{~K}$. 
conductance with respect to $1.5 \mathrm{~K}$, i.e. $\Delta C_{\square}(T)=C_{\square}(T)-C_{\square}(1.5 \mathrm{~K}):$ by this way, we directly compare samples with different conductances. Note that the slope of a $C_{\square}(T)$ curve at low temperature is the important parameter in $2 \mathrm{D}$ localization and not the absolute value of the conductance. We distinguish three régimes which correspond to the régimes of the resistance-fluence characteristic (Fig. 2).

Régime I $\left(R_{\square}(4.2 \mathrm{~K})<300 \Omega\right)$. - The slope of $C_{\square}(T)$ increases with irradiation fluence. This is unexpected since, according to weak localization theory, $\Delta C_{\square}(T)$ should not depend on the atomic disorder.

Régime II $\left(300 \Omega<R_{\square}(4.2 \mathrm{~K})<1000 \Omega\right)$. - The defect concentration is constant and sputtering dominates. The slope is constant in this régime: all samples have the same $\Delta C_{\square}(T)$ curve. This is the expected result, the slope being independent of the film thickness.

At low temperature and in both régimes I and II the conductance increases logarithmically with temperature in agreement with the theory of weak localization in a homogeneous 2D metal (Eq. (6)). A deviation from the logarithmic law is observed at higher temperature and the conductivity maximum, due to the competition between phonons and localization contributions, is shifted to higher temperatures for the more resistive films (see Fig. 5) due to the enhancement of localization.

Régime III. - The holes play the main role. The slope of $C_{\square}(T)$ decreases as the film approaches the percolation threshold : in fact, the experimental variations of the slope follow within $20 \%$ the corresponding modifications of the geometrical form factor $\left(p-p_{\mathrm{c}}\right)^{1.3}$ (Eq. (4)).

We now turn to the temperature dependence of the conductance in régime III (inhomogeneous films). Figure 6 shows the $R_{\square}(4.2 \mathrm{~K})$-fluence dependence for a Pd film irradiated at $4.2 \mathrm{~K}$. Régime III extends over 3 orders of magnitude and can be divided into two parts $\mathrm{A}$ and $\mathrm{B}$.

In $A\left(R_{\square}(4.2 \mathrm{~K})<10^{6} \Omega\right)$, the samples exhibit metallic behavior : in the temperature range $100 \mathrm{mK}<T<1 \mathrm{~K}$ the conductance follows the usual logarithmic law and in the range $1 \mathrm{~K}<T<20 \mathrm{~K}$ it follows a negative power law (Fig. 7) :

$$
C_{\square}(T) \approx T^{-0.27}
$$

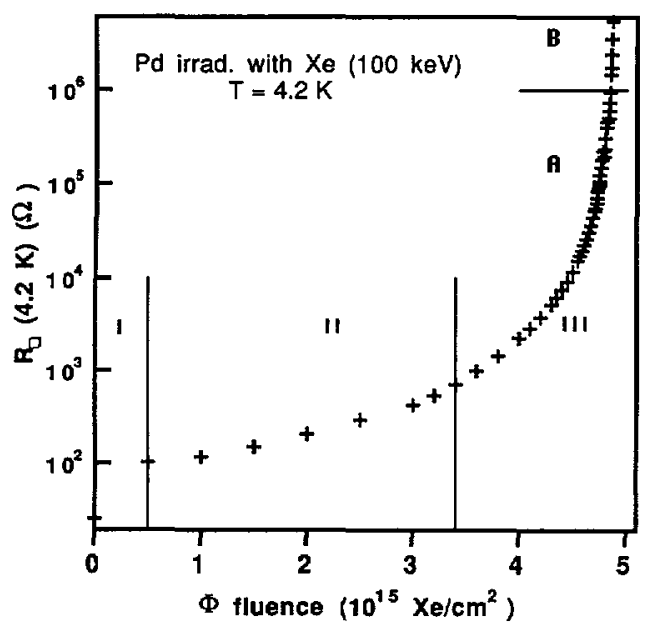

Fig. 6. - 4.2 K resistance-fluence curve. The régime $\mathrm{II}$ may be separated into two regions, following the resistive behavior of the samples : A) metallic samples and B) insulating samples. 


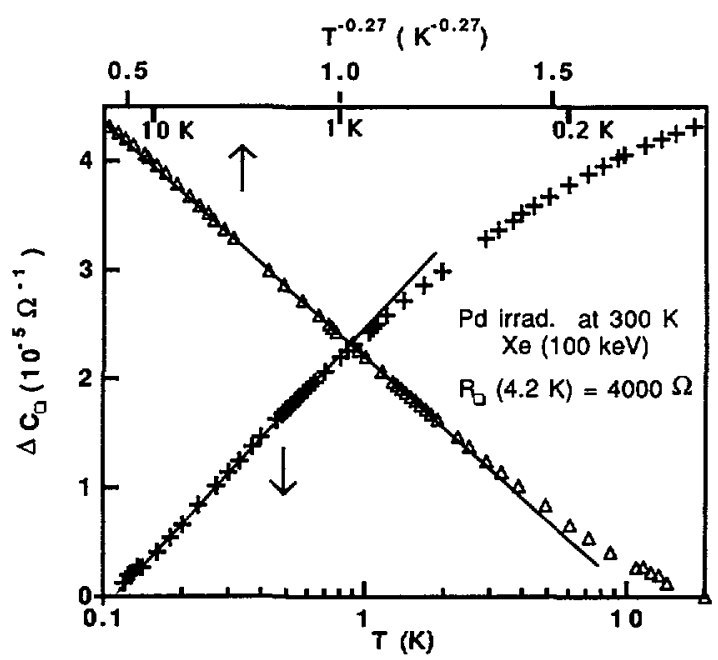

Fig. 7. - Temperature dependence of the conductance of a percolating, metallic film. The experimental points are drawn in a logarithmic temperature scale $(x)$ and a $T^{-0.27}$ scale $(\Delta)$.

The uncertainty on the exponent is less than $10 \%$. We interpret this behavior as a percolation-localization crossover. Two characteristic length scales $L_{\phi}$ and $\xi_{\mathrm{P}}$ are involved in the problem, as explained in chapter 2.3. The analysis of MC data is a natural way to determine $L_{\phi}$. This has been easily done on the non irradiated $\mathrm{Pd}$ but, as we will mention subsequently, a quantitative analysis has not been possible for the irradiated samples. Therefore, we evaluate $L_{\phi}$ for the irradiated films by remembering that, if it is controlled by the inelastic electron-electron interaction $[1,29]$, it varies linearly with the elastic m.f.p. The elastic m.f.p. is directly obtained from the resistivity at $4.2 \mathrm{~K}$ : we deduced $230 \AA$ for the non irradiated films and $80 \AA$ for the irradiated film with saturated bulk disorder. From the MC results, $L_{\phi}(T)$ variation was deduced for the non irradiated films; we then evaluated $L_{\phi}$ for the irradiated sample. An estimate of $\xi_{\mathrm{P}}$ is obtained from the T.E.M. observations. We thus find a ratio $L_{\phi} / \xi_{\mathrm{P}}$ varying from 0.04 to 0.4 in the temperature range investigated here. By comparing the experimental conductance variation with equation (5), we obtain an inelastic collision exponent $\alpha$ equal to 1 . This value was previously found [29] in $\mathrm{Pd}:$ it is due to the dominating electron-electron interaction at low temperature. A similar percolationlocalization crossover was observed on annealed Pd films [17].

In régime $B\left(R_{\square}(4.2 \mathrm{~K})>10^{6} \Omega\right)$, the conductance varies exponentially with temperature (Fig. 8) :

$$
C_{\square}(T) \approx \exp \left(-\frac{T_{0}}{T}\right) .
$$

This relation is a signature of an Anderson insulator, in which conduction occurs via thermally activated hopping between neighbouring localized states. In the régime B, our samples lie just above the percolation threshold $\left(p>p_{c}\right)$ and are weakly disordered at the atomic scale $\left(\ell_{\mathrm{b}}=80 \AA\right)$. The constant $T_{0}$ is related to the extension $\xi_{\ell}$ of a localized state by the equation [24] :

$$
k_{\mathrm{B}} T_{0}=\left(n_{\mathrm{F}} \xi_{\ell}\right)^{-1}
$$

$n_{\mathrm{F}}$ is the density of states at the Fermi level which is non-zero in an Anderson insulator. Using 


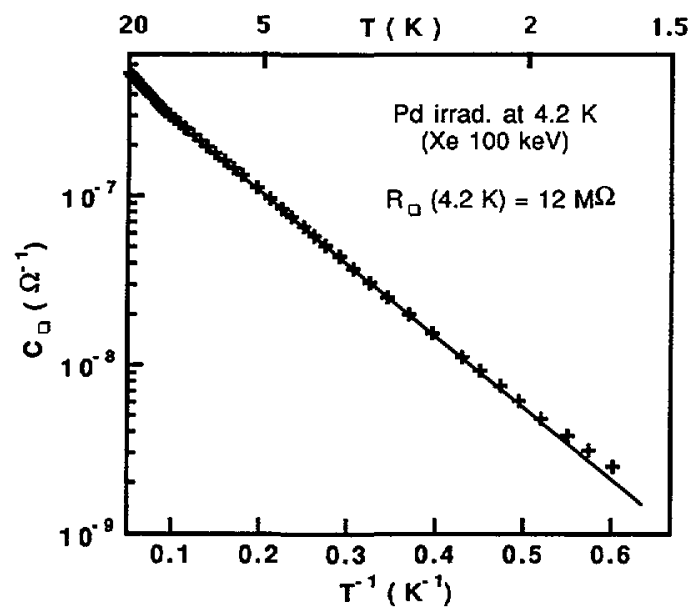

Fig. 8. - Plot of the log of the conductance versus $T^{-1}$ for an insulating sample.

the value of $n_{\mathrm{F}}$ for pure Pd and the experimental value $T_{0}=10 \mathrm{~K}$ we deduce $\xi_{\ell}=50 \AA$, much larger than the interatomic distance. The observed Anderson transition is not exclusively due to macroscopic disorder, since $p>p_{c}$, neither to atomic disorder, since the electronic m.f.p. is metallic. The transition is the signature of the interference between the two kinds of disorder, the macroscopic one amplifying the effects of the microscopic one.

At high temperature $(T>40 \mathrm{~K})$, these samples recover a metallic behavior, the conductance being again proportional to the logarithm of the temperature (Fig. 9). By extrapolating the calculated low temperature values of $L_{\phi}$, we find that for $T>40 \mathrm{~K}, L_{\phi}<50 \AA=\xi_{\ell}$, which is the condition for an Anderson insulator to recover a metallic behavior [28]. In this temperature range, the grain size is about $100 \AA$ according to the T.E.M. results and we are in the homogeneous régime, explaining thus the logarithmic behavior of conductance.

The current-voltage curves of the percolating samples are linear over 4 orders of magnitude with and without magnetic field. Palevski and Deutscher [16] reported strong non-linear effects on their percolating gold films and attributed them to non-uniform dissipation in the vicinity of the weak links of the samples. Such effects do not seem to occur in our films.

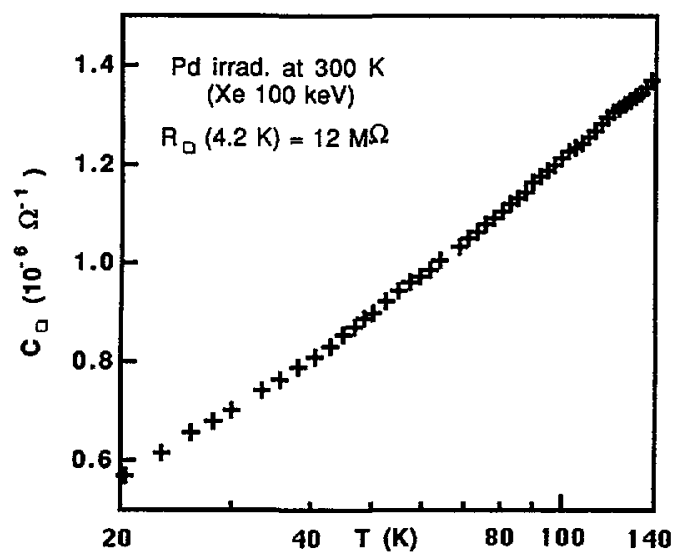

Fig. 9. - Semilog plot of the conductance versus temperature for the insulating sample of figure 8 at high temperature. 


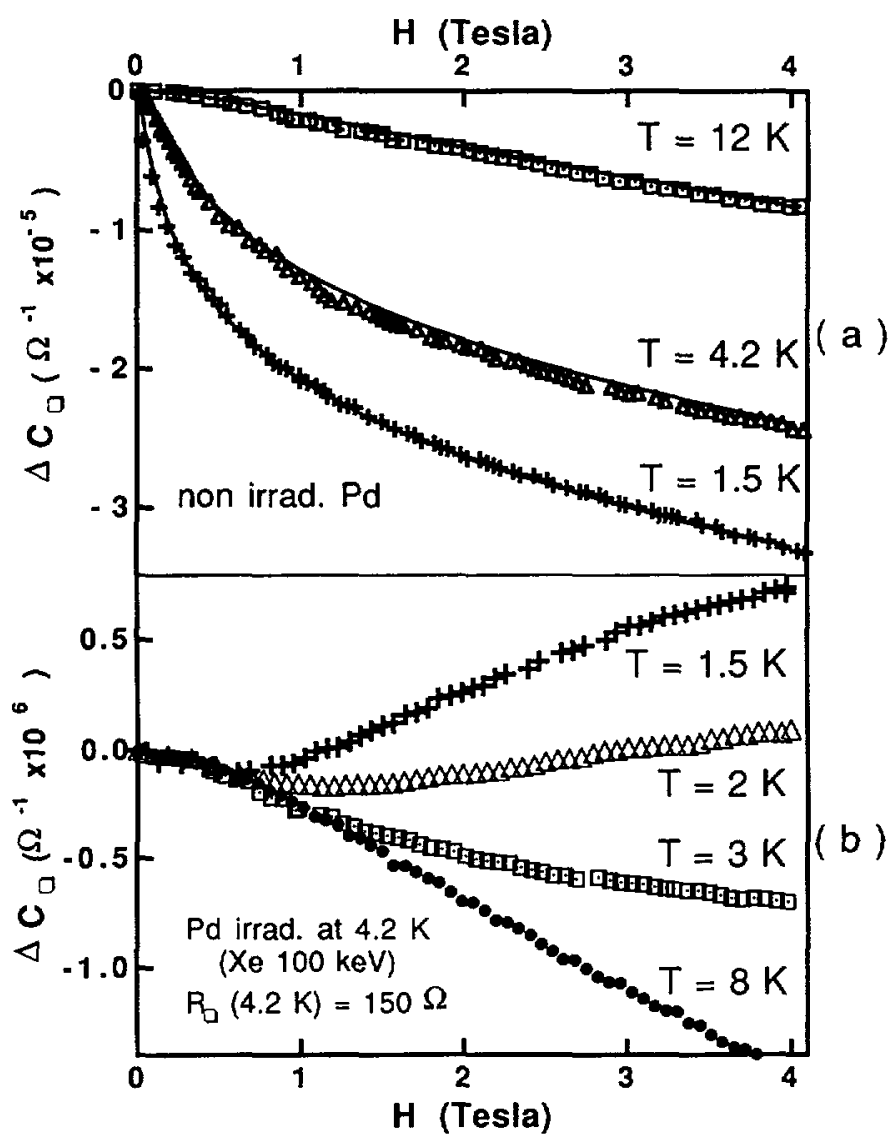

Fig. 10. - Magnetoconductance of the non irradiated Pd (a) and of an irradiated film (b) at the end of régime $\mathrm{I}$.

4.2 MAgneToConduCtanCE. - Figure 10a shows the MC of a non-irradiated $75 \AA$ thick Pd film at different temperatures. The $\mathrm{MC}$ is negative because $\mathrm{Pd}$ is a material with a strong SO interaction [29]. The solid lines correspond to the theoretical variation predicted by weak localization theory [2]. Figure $10 \mathrm{~b}$ shows the $\mathrm{MC}$ of a sample lying at the end of Régime I, i.e. a homogeneous film whose resistivity (and hence atomic defect concentration) has reached saturation. The change in MC is spectacular : it is positive at low temperature and negative at high temperature. The $\mathrm{MC}$ does not change up to the end of régime II. As expected in $2 \mathrm{D}$, the $\mathrm{MC}$ of a sample is independent of its thickness. This behavior persists in the percolating régime III with a decrease in the absolute value of $\mathrm{MC}$ probably related to the existence of holes ; standard localization theory cannot explain the observed MC modifications and we have found no quantitative explanation for this surprising result. A possible cause for this behavior is the fact that $\mathrm{Pd}$ is a metal close to a magnetic ordering which depends on the coordination number [33] and the latter can be modified by irradiation. This possibility is presently under investigation. At the difference of our Pd films, MC measurements on gold films [35] with percolative structure show clearly the role of the anomalous electronic diffusion. 
4.3 THE RESISTANCE-FLUENCE CURVE AT 4.2 K. - Figure 11 shows two resistance-fluence curves : the first (circles) is that of a sample irradiated and measured at $300 \mathrm{~K}$ and the second (crosses) of a sample of the same initial thickness, irradiated and measured at $4.2 \mathrm{~K}$. Notice that the low temperature irradiation allows a close approach of the percolation threshold with stable highly resistive films ; this condition cannot be reached with $300 \mathrm{~K}$ irradiation because the film topology changes with time near the threshold. As we showed that the irradiationinduced disorder is independent of the irradiation temperature (Sect. 3), these two curves are equivalent to a single irradiated film measured at $300 \mathrm{~K}$ and $4.2 \mathrm{~K}$.

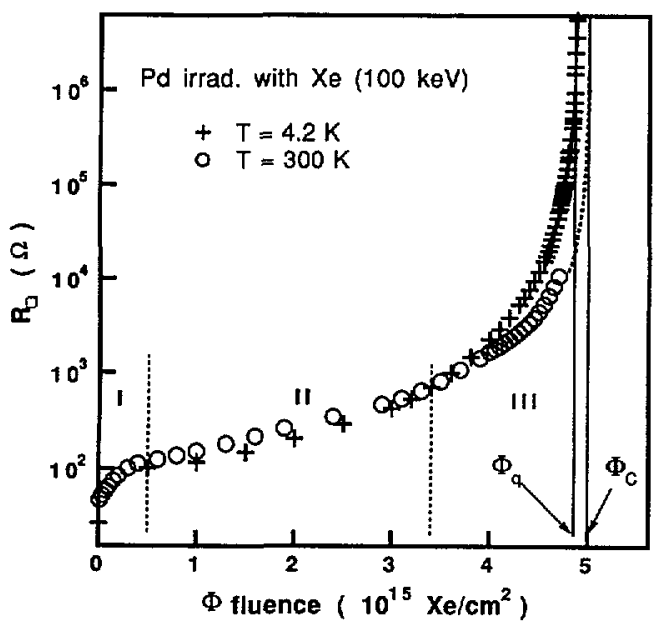

Fig. 11. - Semilog plot of resistance versus fluence for two samples of the same initial thickness, corresponding to an irradiation and resistive measurement at $300 \mathrm{~K}(0)$ and $4.2 \mathrm{~K}(x)$.

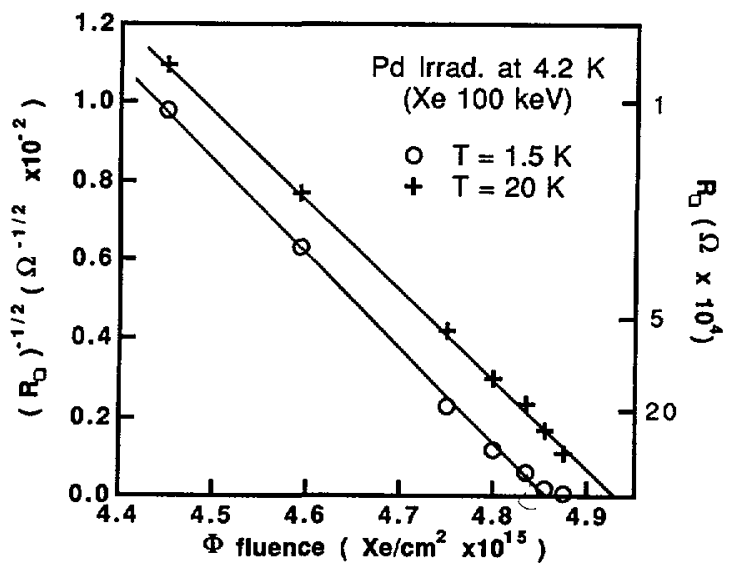

Fig. 12. - Critical behavior of the low temperature resistance at $T=1.5 \mathrm{~K}(\mathrm{O})$ and $T=20 \mathrm{~K}(+)$ for the same sample. The critical exponent is 2 and the critical fluence $\Phi_{\mathrm{q}}$ increases with temperature.

The two resistance-fluence curves behave differently in régime III : $R_{\square}(4.2 \mathrm{~K})$ diverges at a fluence $\Phi_{\mathrm{q}}$ smaller than the percolation fluence $\Phi_{\mathrm{c}}$ determined at $300 \mathrm{~K}$. $\Phi_{\mathrm{q}}$ is not a constant, but increases with increasing temperature (Fig. 12). Moreover, the critical behavior is 
different at low temperature and we observe :

$$
R_{\square}(4.2 \mathrm{~K}) \sim\left(\Phi_{\mathrm{q}}-\Phi\right)^{-2}
$$

The exponent value is different from 1.3, the room temperature value. We conjecture that $\Phi_{\mathrm{q}}$ tends to $\Phi_{\mathrm{c}}$ and the exponent 2 to 1.3 as the temperature goes from $4.2 \mathrm{~K}$ to $300 \mathrm{~K}$. This can be qualitatively understood : at low temperature, quantum localization effects dominate and their interference with the percolating geometry gives rise to a divergence of the resistance above the percolation threshold which is the reflection of the crossover from the metallic to the insulating régime. At $300 \mathrm{~K}$, there are no localization effects and the resistance is only sensitive to the topology of the samples, thus to the percolation geometry.

\section{Conclusion.}

In this paper, we demonstrated the interference between microscopic and macroscopic disorder, both created by irradiation, in thin Pd films. The study of the resistance at $300 \mathrm{~K}$ allowed us to analyze the creation of a percolating structure, while the low temperature resistance involves localization effects which are found to be strongly affected by the inhomogeneous (fractal) nature of the samples.

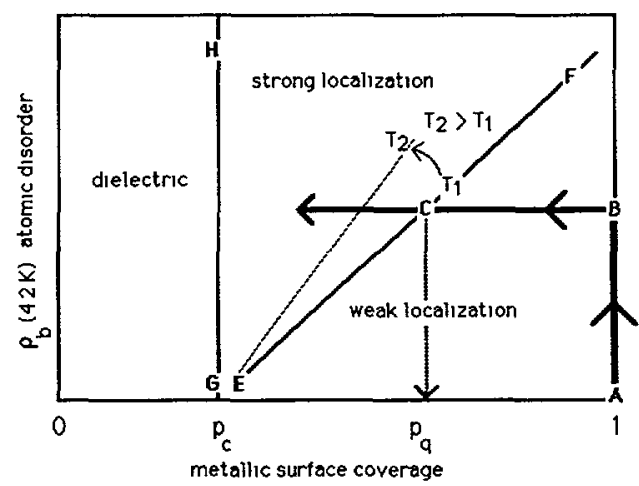

Fig. 13. - Phase diagram for the electrical properties of the irradiated films. The arrows show the evolution of the sample with increasing fluence.

Finally, we propose a phenomenological phase diagram (Fig. 13), based on our results. This kind of diagram has been introduced by Khmelnitskii [9]. It consists of a plot of the bulk resistivity, related to the atomic disorder, versus the surface coverage, related to the macroscopic disorder. The arrows show the evolution of our films with increasing irradiation fluence. The non-irradiated sample is on $A$. The regime $I$ is a line parallel to the vertical axis : $\rho_{\mathrm{b}}$ increases and $p$ remains equal to 1 . Régime II is represented by a single point in the diagram : $\rho_{\mathrm{b}}$ and $p$ are constant. Régime III is a line parallel to the horizontal axis. Along the line $\mathrm{AB}$, the electrical transport is interpreted with the theory of $2 \mathrm{D}$ localization in a homogeneous system. From $B$ to $C$, the behavior is still metallic, but modified by the percolating geometry of the metallic conducting cluster. This is the region of the crossover from the logarithmic to the power law temperature dependence of the conductance. Once point $\mathrm{C}$ is reached, the Anderson transition is crossed and we obtain the exponential laws. The transition line EF is temperature-dependent: it moves to the left with increasing temperature, because more disorder is needed to cross the Anderson transition. At 
sufficiently high temperature, EF merges with the vertical line $\mathrm{GH}$, which describes the percolation transition at $p_{c}$. As EF moves to the left with increasing temperature, point $\mathrm{C}$ is found on the metallic side of the transition. This corresponds to the temperature-induced Anderson transition, characterized by the crossover from an exponential law at low temperature to a logarithmic law at higher temperature.

\section{Acknowledgements.}

H. Bernas, L. Dumoulin, J. Lesueur, J. Rosenblatt and A. Traverse are acknowledged for many fruitful discussions. We are especially grateful to L. Dumoulin for film evaporations, A. Traverse for the R.B.S. experiments, M. O. Ruault for the T.E.M. pictures, O. Kaitasov for assistance with the irradiations and A. Benot for his efficient help in the very low temperature experiments.

\section{References}

[1] Lee P. A. and Ramakrishnan T. V., Rev. Mod. Phys. 57 (1985) 287.

[2] Bergmann G., Phys. Rep. 107 (1984) 1.

[3] Altshuler B. L. and Aronov A. G., in Electron-Electron Interaction in Disordered Conductors, A. L. Efros and M. Pollak Eds. (North Holland, Amsterdam, 1985) p. 1.

[4] STAuffer D., Introduction to Percolation Theory (Taylor and Francis, London and Philadelphia, 1985).

[5] Deutscher G., in Chance and Matter, J. Souletie, J. Vannimenus and R. Stora Eds. (North Holland, Amsterdam, 1987) p. 1.

[6] Landauer R., in Electrical Transport and Optical Properties of Inhomogeneous Media, J. C. Garland and D. B. Tanner Eds. (American Institute of Physics, New York, 1978) p. 2.

[7] Toulouse G., C. R. Acad. Sci. B 289 (1975) 629.

[8] Friedel J., J. Phys. 15 (1975) 655.

[9] KhMELNITSKII D. E., JETP Lett. 32 (1980) 229.

[10] Gefen Y., Thouless D. J. and Imry Y., Phys. Rev. B 28 (1983) 6677.

[11] ShapIRo B., in Percolation, Structure and Processes, G. Deutscher, R. Zallen and J. Adler Eds. (Israel Physical Society, Jerusalem, 1983) p. 367.

[12] LÉVY Y. and SOUILlaRd B., Europhys. Lett. 4 (1987) 233.

[13] Deutscher G., LÉvy Y. and Soulllard B., Europhys. Lett. 4 (1987) 577.

[14] Deutscher G., Goldman A. M. and Micklitz H., Phys. Rev. B 31 (1985) 1679.

[15] Nédellec P., Traverse A., Dumoulin L., Bernas H., Amaral L. and Deutscher G., Europhys. Lett. 2 (1986) 465.

[16] Palevski A. and Deutscher G., Phys. Rev. B 34 (1986) 431.

[17] Papandreou N. and Nédellec P., Physica B 165\&166 (1990) 281.

[18] Papandreou N., Nédellec P. and Traverse A., Solid State Commun. 73 (1990) 285.

[19] Papandreou N., NÉdellec P. and Rosenblatt J., Appl. Phys. Lett. 54 (1989) 537.

[20] Bergmann (G.), Phys. Rev. B 35 (1987) 4205.

[21] Prober D. E., in Percolation, Localization and Superconductivity, A. Goldman and S. Wolf Eds. (Plenum Press, London, 1984) p. 231.

[22] Schmid A., in Localization, Interaction, and Transport Phenomena, B. Kramer, G. Bergmann and Y. Bruynseraede Eds., Solid State Sci. 61 (Springer-Verlag, Berlin, 1984) p. 212.

[23] Hikami S., Larkin A. I. and Nagaoka Y., Progr. Theor. Phys. 63 (1980) 707.

[24] MotT N., Metal-Insulator Transitions (Taylor and Francis, London, 1974).

[25] Miller A. and Abrahams E., Phys. Rev. 120 (1960) 745.

[26] Ambegaokar V., Halperin B. I. and Langer J. S., Phys. Rev. 4 (1971) 2612.

[27] Efros A. L. and Shklovskil B. I., J. Phys. Colloq. France 36 (1975) C8-L49. 
[28] Souillard B., in Chance and Matter, J. Souletie, J. Vannimenus and R. Stora Eds. (North Holland, Amsterdam, 1987) p. 305.

[29] Raffy H., Nédellec P., Dumoulin L., Maclachlan D. S. and Burger J. P., J. Phys. France 46 (1985) 627.

[30] Traverse A., Paumier E., Nédellec P., Bernas H., Dumoulin L. and Chaumont J., Phys. Rev. B 37 (1988) 2495.

[31] Sondheimer E. H., Adv. Phys. 1 (1952) 1.

[32] Sigmund P., in Inelastic Ion-Surface Collisions, N. H. Tolk, J. C. Tully, H. Heiland and C. W. White Eds. (Academic Press, New York, 198) p. 121.

[33] Fink R. L., Ballentine C. A., Erstein J. L. and Araya-Pochet J. A., Phys. Rev. B 41 (1990) 10175 .

[34] Seeger A., Schumacher D., Schilling W. and Diehl J. Eds., Vacancies and Interstitials in Metals (North Holland, Amsterdam, 1970).

[35] Dumpich G. and Carl A., Phys. Rev. B 43 (1991) 12074. 